\title{
Análise envoltória de dados para aprimorar a sistemática de precificação em um restaurante casual dining
}

\author{
RESUMO
}

\section{Giovanni Susin}

gbsusin@gmail.com

Universidade Federal do Rio Grande do Sul (UFRGS), Porto Alegre, Rio Grande do Sul, Brasil

Michel José Anzanello

michel.anzanello@gmail.com

Universidade Federal do Rio Grande do Sul (UFRGS), Porto Alegre, Rio Grande do Sul, Brasil

\section{Alessandro Kahmann} alessandrokahmann@hotmail.com Universidade Federal do Rio Grande do Sul (UFRGS), Porto Alegre, Rio Grande do Sul, Brasil

\section{Lucas Bogdanov Schmidt}

lucasximiti@hotmail.com

Universidade Federal do Rio Grande do Sul (UFRGS), Porto Alegre, Rio Grande do Sul, Brasil
A intensa competitividade nos mais diversos ramos de atuação incita as empresas a encontrarem soluções para se diferenciar da concorrência e prosperar no mercado. No setor de alimentação a lucratividade se relaciona diretamente com os preços de seus produtos, tornando a precificação importante no planejamento estratégico da empresa. Situado dentro deste cenário, este estudo propõe a utilização da Análise Envoltória de Dados (AED) para o desenvolvimento de uma sistemática de precificação em um restaurante casual dining. Inicialmente foram gerados índices que descrevem a eficiência de cada prato em utilizar os recursos do restaurante. Com base nesses índices, os pratos menos eficientes foram reajustados, tendendo a receber um preço maior. Na sequência, foram simulados cenários de custos pela equipe de precificação do restaurante, avaliandose assim o impacto da utilização do índice na determinação dos preços. Os resultados obtidos mostram que o método desenvolvido foi eficiente em incluir na sistemática de precificação fatores outros que o custo de ingredientes.

PALAVRAS-CHAVE: Engenharia de cardápio. Precificação. Análise envoltória de dados. Restaurante casual dining. Seleção de variáveis. 


\section{INTRODUÇÃO}

$\mathrm{O}$ atento monitoramento dos custos operacionais em ambientes de intensa concorrência é uma necessidade comum a organizações dos mais diversos setores. De tal forma, a manutenção da lucratividade passa a ter relação direta com os preços, cuja definição torna-se tarefa fundamental para a estratégia de qualquer negócio. Buscando sistematizar o processo de precificação, diversas ferramentas foram propostas para a ponderação de fatores econômicos e produtivos na determinação dos preços (SOON, 2011). No setor de alimentação não é diferente: a precificação impacta diretamente na viabilidade do negócio, justificando o desenvolvimento de técnicas para a sua composição.

As técnicas normalmente utilizadas para a composição de cardápios apoiamse em matrizes com quatro quadrantes para a classificação dos pratos de acordo com a sua margem de contribuição (preço subtraído do custo com alimentos) e a popularidade do item. Miller (1980) foi o primeiro a desenvolver o modelo matricial, utilizando a margem de contribuição em percentual e a popularidade do prato para analisar o desempenho de cada item. Já Kasavana e Smith (1982) utilizaram a análise de portfolio do Boston Consulting Group como base para a criação do conceito de ME (Engenharia de Cardápio), que incorporou à análise de Miller a utilização da margem bruta em unidades monetárias. Pavesic (1983), por sua vez, uniu os dois modelos desenvolvendo o modelo de Custo/Margem, que considera a margem bruta de cada item em unidades monetárias, ponderada pela quantidade de produtos vendidos. Assim, os produtos passam a ser classificados pela sua capacidade de gerar lucro para o restaurante.

Esses modelos, apesar de serem de grande utilidade para a composição dos cardápios, possuem uma grande deficiência: não consideram os custos operacionais nas análises (RAAB; MAYER, 2007). Assim, buscando tornar os modelos mais confiáveis, outras técnicas foram desenvolvidas para complementar a análise de cardápio, destacando-se recentes aplicações do Custeio Baseado em Atividades ( $A B C$ ) para a ponderação dos custos indiretos (RAAB; MAYER, 2007; LINASSI, 2009). No entanto, segundo Taylor et al. (2009), a utilização do $A B C$ ainda é um desafio, pois requer medições constantes dos custos e se baseia em estimativas para analisar o desempenho de pratos que ainda não fazem parte do cardápio. Como alternativa, Taylor et al. (2009) propõem a utilização da Análise Envoltória de Dados (AED), ferramenta que gera um índice de eficiência com base no impacto das variáveis de entrada (inputs) sobre as variáveis de saída (outputs). Com a utilização da AED, múltiplos fatores são integrados à análise matricial, tornando a mesma mais confiável ao incorporar custos não considerados nas abordagens tradicionais de engenharia de cardápio. Posteriormente, o trabalho de Taylor et al. (2009) foi validado através do uso da modelagem por equações estruturais, confirmando a eficiência na utilização da AED na estruturação de cardápios (REYNOLDS; TAYLOR, 2011).

Este artigo propõe uma sistemática baseada na AED para criar índices de eficiência que auxiliem na determinação dos preços de um novo cardápio. 0 modelo é desenvolvido através de um estudo de caso realizado com base nos dados de duas unidades de uma rede de restaurantes casual dining, localizadas no Sul do Brasil. Tais estabelecimentos utilizam o método de custo/margem proposto por Pavesic (1983) para determinação dos produtos que permanecerão no cardápio e posterior precificação dos mesmos. Essa análise, porém, não 
pondera outros fatores que impactam tanto em custos como na qualidade do serviço prestado ao cliente. Pratos de preparo menos complexo, por exemplo, poderiam ter a venda incentivada através da redução do preço, visto que oneram menos trabalho da cozinha, são de preparo mais rápido e geram menor desperdício. No entanto, a dificuldade está em determinar o impacto de tais fatores em cada prato e o quão significativo é essa influência na lucratividade do restaurante.

A sistemática aqui proposta inicia com o levantamento das variáveis capazes de descrever o impacto que a elaboração de cada prato gera nos fatores de produção do restaurante. Essa etapa é realizada através do mapeamento dos processos da cozinha e de entrevistas com a equipe gerencial. Na sequência, tais variáveis são selecionadas em termos de sua não redundância. Em seguida, a AED é utilizada para avaliar a eficiência de cada prato com base nas variáveis selecionadas. Por fim, são criados índices em valor monetário que, somados ao custo dos ingredientes de cada prato, incorporam os fatores de produção no método de precificação.

\section{REFERENCIAL TEÓRICO}

Esta seção é composta por dois temas: Análise Envoltória de Dados (AED) e Precificação de Cardápios. Inicialmente é feita uma introdução à $A E D$, onde são apresentados os objetivos e aparato matemático da ferramenta.

\section{ANÁLISE ENVOLTÓRIA DE DADOS}

A Análise Envoltória de Dados é uma ferramenta utilizada para medição e avaliação do desempenho de entidades chamadas Unidades Tomadoras de Decisão (Decision-Making Units - DMUs), que convertem múltiplas variáveis de entrada (inputs) em múltiplas variáveis de saída (outputs) (COOPER et al., 2011). No estudo que deu origem ao conceito de DMU, Charnes et al. (1978) definiram a AED como um modelo de programação matemática aplicado para a estimativa empírica de relações que são pilares da economia moderna, como as funções de produção.

Apesar do nome, as DMUs não necessariamente são unidades que tomam decisões. Belloni (2000) considerou instituições de ensino como as DMUs em seu artigo que avaliou o desempenho das mesmas. Já Taylor et al. (2009) consideraram como DMUs os pratos servidos por um restaurante, comparando a eficiência dos mesmos em gerar lucro para o estabelecimento. Uma vez que a definição de DMU é genérica e flexível, a AED vem sendo aplicada como ferramenta de análise de eficiência nos mais diversos setores e contextos (REYNOLDS; THOMPSON, 2007).

Mello et al. (2005) colocam como objetivo primário da AED comparar um certo número de DMUs que realizam tarefas similares, diferenciando-se nas quantidades de saída produzidas para cada nível de recurso consumido. Os autores destacam também os seguintes objetivos da AED: 
a) estimar uma função de produção linear por partes (piece-wise linear frontier) das DMUs eficientes que possa servir como referência para avaliar as DMUs ineficientes;

b) determinação da eficiência relativa das DMUs, avaliando o quanto cada DMU é eficiente em relação às DMUs eficientes. Essa análise permite que a AED seja utilizada como uma ferramenta de apoio à decisão já que pode estabelecer uma relação de ordem entre as DMUs;

c) elaborar estratégias de produção que possam maximizar a eficiência das DMUs avaliadas, corrigindo os fatores de entrada que geram as ineficiências;

d) apoiar decisões gerenciais com o estabelecimento de taxas de substituição entre as entradas, entre as saídas e entre entradas e saídas;

e) considerar a possibilidade de os outliers não serem apenas desvios em relação à média, mas sim unidades que adotam melhores práticas.

Os outliers podem, portanto, servir como referência para melhorar as demais DMUs; (vi) Estimar a fronteira produtiva sem a necessidade de uma forma funcional, como ocorre nos modelos de Fronteira Estocástica (AIGNER et al., 1977; MEEUSEN; VAN DEN BROECK, 1977).

\section{Definições}

O conceito de eficiência, fundamental para o entendimento da AED, é um conceito relativo, pois compara o que foi produzido, dado os recursos disponíveis, com o que poderia ter sido produzido com os mesmos recursos (MELLO et al., 2005). Na Análise Envoltória de Dados, considera-se que uma DMU é $100 \%$ eficiente quando apresentar uma relação ótima de transformação de inputs em outputs, ou seja, quando não for possível melhorar o desempenho da DMU sem que seja piorado o desempenho de outra DMU. Por essa definição, não é necessária a determinação de preços ou pesos para refletir a importância dessas variáveis, assim como se evita também a necessidade de especificar explicitamente as relações formais que supostamente existem entre elas (COOPER et al., 2011).

Existem duas formas básicas para tornar eficiente uma unidade não eficiente. É possível reduzir os recursos, mantendo constantes os produtos (orientação a inputs), ou então o inverso (orientação a outputs). Os níveis máximos de produção obtidos para cada nível de recurso, por sua vez, formam uma curva denominada Fronteira de Eficiência, sendo a região abaixo dessa curva chamada de Curva Viável de Produção. Esses conceitos são exemplificados na Figura 1, em que o eixo $X$ representa os recursos utilizados e o eixo $Y$ a produção. A curva $S$, Fronteira de Eficiência, indica o máximo que foi produzido com cada tipo de recurso. Os pontos $C$ e $B$ são considerados eficientes, uma vez que se encontram sobre a Fronteira de Eficiência. O ponto A, por sua vez, ilustra uma unidade não eficiente, porém localizada no chamado Conjunto Viável de Produção, área abaixo da curva S. Existem duas maneiras de tornar $\mathrm{A}$ eficiente: redução dos recursos de entrada necessários (inputs), equivalente ao deslocamento do ponto $\mathrm{A}$ até o ponto $\mathrm{C}$, ou aumento da produção (outputs) 
mantendo-se os recursos, que equivale a deslocar o ponto $\mathrm{A}$ até o ponto $\mathrm{B}$ (MELLO et al., 2005).

Figura 1 - Produtividade X eficiência

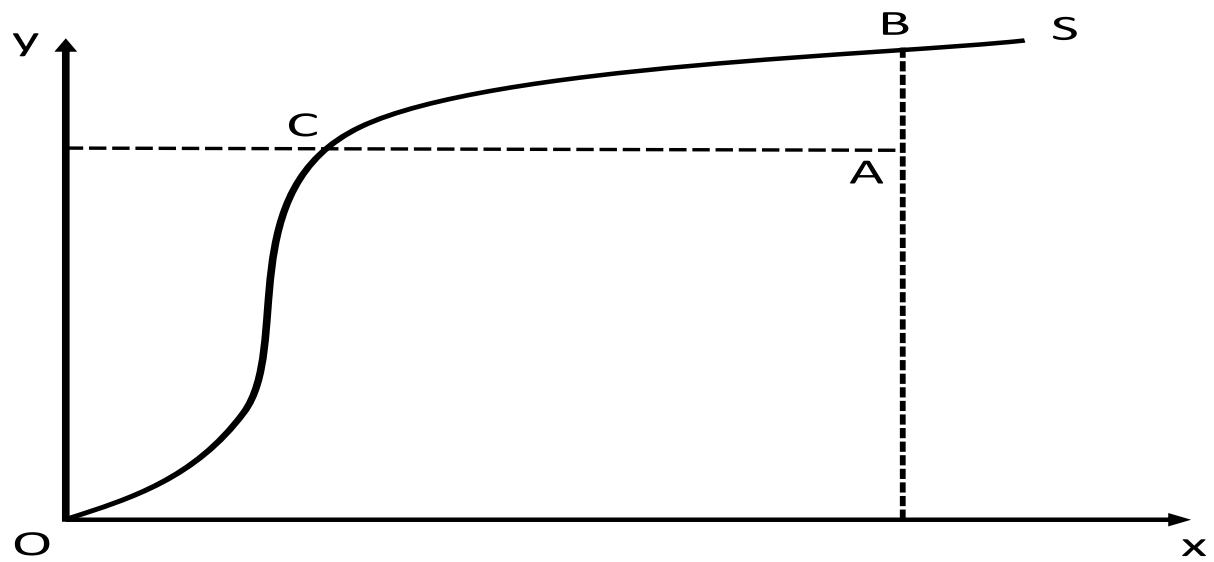

Fonte: Adaptado de Mello et al. (2005).

\section{Modelo CCR}

Um dos modelos clássicos para aplicação da Análise Envoltória de Dados foi apresentado por Charnes et al. (1978), que sugeriram o Modelo CCR (Modelo de Charnes, Cooper e Rhodes) para avaliação das atividades de entidades sem fins lucrativos (DMUs) participantes de programas públicos. Esse modelo foi concebido inicialmente com orientação a entradas (inputs), e trabalha com retorno constante de escala, ou seja, variações nos inputs provocam variações proporcionais nos outputs (Charnes et al., 1978).

No modelo CCR, a relação dos inputs e outputs é utilizada para medir a eficiência relativa da $\mathrm{DMU}_{j}=\mathrm{DMU}_{0}$, a ser avaliada com base nas relações de todas as $j=1,2, \ldots, n \mathrm{DMU}_{j}$. Considera-se que existem $n$ DMUs a serem avaliadas e que cada uma dessas DMUs consome diferentes quantidades de $m$ diferentes inputs para produzir $s$ diferentes outputs. Em termos mais específicos, cada $\mathrm{DMU}_{j}$ consome uma quantidade $x_{i j}$ do input $i$ (a mão de obra em horas/produto utilizada, por exemplo) e produz uma quantidade $y_{r j}$ do output $r$ que poderia ser, nesse caso, a quantidade de produtos/hora. Assume-se também que $x_{i j}$ e $y_{r j}$ são maiores ou iguais a zero, e que cada DMU possui pelo menos um input e um output positivos (COOPER et al., 2011).

Com as variáveis definidas, inicia-se a determinação das relações que compõem a AED. Para facilitar a formulação do problema de programação linear, o modelo CCR pondera todos os inputs em apenas uma variável, procedimento também realizado para os outputs. A construção do modelo CCR pode ser interpretada, portanto, como a redução de múltiplos inputs/outputs existentes para cada DMU a um cenário no qual se considera apenas um input e um output virtuais. 
$\mathrm{Na}$ linguagem de programação matemática, essa relação pode ser representada conforme a equação (1). Nessa equação, as variáveis são os $u_{r} s$ e os $v_{i} s$ sendo que os $y_{r o} s$ e os $x_{i o} s$ são os observados de input e output, respectivamente, para a $\mathrm{DMU}_{\text {o }}$ que está sendo avaliada. O modelo CCR propõe ainda restrições para que a equação represente a condição em que os inputs e outputs virtuais de cada $\mathrm{DMU}$, incluindo $\mathrm{DMU}_{j}=\mathrm{DMU}_{0}$, seja menor ou igual a uma unidade, gerando o seguinte modelo de programação fracionário:

$$
\max _{h o}(u, v)=\frac{\sum_{\mathrm{r}} u_{r} y_{r o}}{\sum_{i} v_{i} x_{i o}}
$$

Sujeito a:

$$
\begin{gathered}
\frac{\sum_{r} u_{y} y_{r j}}{\sum_{i} v_{i} x_{i j}} \leq 1 \text { para } j=1, \ldots . ., n . \\
u_{r} v_{i} \geq 0 \text { para todo } i \text { e } r
\end{gathered}
$$

O modelo acima pode gerar um número infinito de soluções. Sendo ( $\left.u^{*}, v^{*}\right)$ soluções ótimas, então $\left(\alpha u^{*}, \alpha v^{*}\right)$ serão também ótimos para todo $\alpha>0$. Uma vez que é necessário determinar os valores dos pesos $u_{j}$ e $v_{i}$ para maximizar a soma dos outputs, Charnes e Cooper (1962) desenvolveram uma transformação do modelo CCR para um modelo de programação linear equivalente. A mudança das variáveis $(u, v)$ para $(\mu, v)$ é resultado dessa transformação:

$$
\theta^{*}=\min \theta(2)
$$

Sujeito a:

$$
\begin{gathered}
\sum_{j=1}^{n} x_{i j} \gamma_{j} \leq \theta x_{i o} \quad i=1,2, \ldots, m \\
\sum_{j=1}^{n} y_{i j} \gamma_{j} \leq \theta y_{r o} \quad r=1,2, \ldots, s \\
\gamma_{j} \geq 0 j=1,2, \ldots, n .
\end{gathered}
$$

\section{Aplicações da Análise Envoltória de Dados}

A Análise Envoltória de Dados tem sido aplicada para a avaliação de DMUs em diferentes setores. Pioneiros na aplicação, Charnes et al. (1978) utilizam a mesma para a avaliação de organizações sem fins lucrativos e dão ênfase para o uso da ferramenta no setor público. Na pesquisa, escolas são vistas como DMUs e os outputs são as médias aritméticas dos estudantes, testes psicológicos realizados com os mesmos e a capacidade dos alunos de entender movimentos do corpo. Como inputs, considera-se valores como o número de horas professor, o tempo gasto pelos pais com os programas, entre outros. $O$ estudo gerou uma série de meios para se medir a eficiência em programas públicos, objetivando-se planejar e controlar essas atividades.

As aplicações da AED, porém, não se restringem ao setor público. Mello et al. (2003) utilizaram a ferramenta para avaliar a eficiência das companhias aéreas brasileiras. A análise foi dividida em três partes, avaliando os aspectos operacionais, comerciais e globais de cada companhia. Como resultados, foram descobertas eficiências não evidentes em algumas companhias, assim como a explicação de algumas ineficiências. 
No setor de hospitalidade estudos também se apoiam na Análise Envoltória de Dados para avaliar operações similares. Hsieh e Lihn (2010) utilizaram a DEA para construir um modelo que permitisse a avaliação da eficiência de hotéis internacionais em Taiwan. O modelo avaliou o desempenho de diferentes departamentos dos hotéis e resultou em uma média de eficiência utilizada para avaliar quais setores interferiam negativamente ou positivamente em cada hotel. Reynolds e Thompson (2007), por sua vez, avaliaram os efeitos de variáveis não controláveis em unidades de uma rede de restaurantes nos Estados Unidos. Concluiu-se que apenas $12 \%$ das unidades operavam de maneira $100 \%$ eficiente e que a média de eficiência da rede era de $82 \%$.

Ainda no setor de hospitalidade, também foram desenvolvidos estudos que utilizaram a DEA para avaliar a composição de cardápios. Chou e Fang (2012) analisaram o desempenho dos pratos principais de uma cadeia de restaurantes chineses fast-food. Custos de mão-de-obra e despesas operacionais indiretas foram incorporados à análise de engenharia de cardápio para auxiliar os gerentes a manter, incentivar ou desestimular a venda de determinados pratos durante alterações sazonais. Os resultados obtidos mostram que o desempenho financeiro das unidades tende a ser melhor quando utilizado o modelo que incorporou a AED à análise de cardápio. Esse mesmo resultado foi obtido no estudo desenvolvido por Taylor et al. (2009), que também incorporaram a AED às abordagens de análise de cardápio tradicionais. Foram avaliados fatores como custos com mão-de-obra, número de ingredientes do prato, validade dos ingredientes, entre outros, como variáveis de entrada para avaliar o impacto delas na margem bruta e na popularidade dos itens (outputs).

\section{PRECIFICAÇÃO DE CARDÁPIOS}

A precificação do cardápio influencia diretamente o número de clientes e seus gastos no restaurante. Assim, o preço deve não só contribuir para o aumento da lucratividade do restaurante, como também parecer razoável sob o ponto de vista dos clientes (PAVESIC, 1989). A otimização desses preços, porém, ainda é um desafio para os administradores de restaurantes, visto que não se está no cenário clássico da economia em que o preço é definido pelo mercado. Os concorrentes oferecem pratos semelhantes, porém não exatamente iguais, e a diferenciação ocorre por critérios como a qualidade, decoração e serviço prestado, entre outros (KIEFER et al., 1994). Além disso, o consumidor considera os valores dos demais pratos do cardápio antes de optar pelo seu, fazendo com que os pratos também concorram entre si (Kelly et al., 2009).

Buscando entender essa sistemática de determinação de preços de um cardápio, realizam-se estudos tanto sob o ponto de vista quantitativo como abordagens focadas nos fatores psicológicos da relação entre o cliente e o cardápio. Kelly et al. (2009) avaliaram a elasticidade da demanda de um determinado prato do cardápio. Foram distribuídos cardápios com preços diferentes aos clientes e o resultado foi que a demanda pelo prato não diminuiu com aumentos razoáveis dos valores. Tal estudo, no entanto, não considera a possibilidade do cliente substituir o prato em questão por outro semelhante, porém sinaliza que, em algumas situações, pode haver espaço para aumentos de preços sem prejudicar a demanda. 
Hayes e Huffman (1995), por sua vez, avaliaram o impacto que descontos em determinados produtos podem ter na lucratividade de restaurantes. $O$ estudo concluiu que essa prática tem grande potencial para aumentar os ganhos, porém deve ser aplicada junto a forte investimento em publicidade, sendo indicada para grandes redes. Também com enfoque na melhoria da lucratividade, Alvarez et al. (2011) propõem um modelo de otimização para auxiliar no reajuste de preços nos cardápios; tal abordagem determina a frequência ideal de reajustes, comparando o custo de reajuste com a perda do não reajuste.

Já o estudo de Pavesic (1989) elenca uma série de fatores psicológicos que influenciam na precificação de cardápio. Segundo o autor, pratos com os preços mais altos passam ao cliente a percepção de maior qualidade, independente do custo de produção dos mesmos. $O$ autor também coloca que a presença de pratos de valor mais elevado no cardápio altera a percepção do valor dos demais pratos, fazendo com que os mesmos pareçam "barganhas".

Na mesma linha de pesquisa, Carmin et al. (1990) citam que a demanda por um prato principal não varia com alterações que não modificam o digito principal (apenas nos centavos). $\mathrm{O}$ autor coloca ainda que o preço do prato principal mais caro não deve nunca ser maior do que 2,5 vezes o preço do prato principal mais em conta. Já Yang et al. (2009) encontraram diferenças também no modo como o preço é apresentado ao cliente. Segundo o autor, o uso do cifrão ao lado do valor influencia negativamente a demanda por um item, ao passo que a apresentação do valor em numerais ou escrito por extenso não altera a percepção dos clientes.

\section{PROCEDIMENTOS METODOLÓGICOS}

O presente estudo apoia-se em uma pesquisa que aplica métodos quantitativos, através de um estudo de caso, para a solução de um problema. Assim, pode ser considerada uma pesquisa exploratória de natureza aplicada e abordagem quantitativa (GIL, 2008). A aplicação do estudo de caso ocorre em duas unidades de uma rede de restaurantes localizadas no Sul do Brasil. Cada loja serve, respectivamente, uma média de 16000 e 8000 refeições/mês, e trabalha com o conceito casual dining, segundo o qual pratos variados são servidos em um ambiente casual e acolhedor, tornando a qualidade do serviço o ponto chave do negócio.

Os estabelecimentos funcionam na modalidade de franquias, devendo seguir padrões de atendimento e produção dos pratos formulados pela franqueadora. Não é possível, portanto, inserir ou remover pratos do cardápio, alterar suas composições ou até mesmo a sequência de produção dos mesmos sem que haja autorização da franqueadora. A precificação dos cardápios, no entanto, tem liberdade para ser executada localmente e é realizada duas vezes ao ano, quando ocorrem as trocas de cardápios.

Com o objetivo de aprimorar esse processo de precificação, foi desenvolvido o método que será detalhado a seguir. A estruturação do método foi baseada na pesquisa desenvolvida por Taylor et al. (2009), e é dividida em quatro etapas, conforme ilustra a Figura 2. 
Figura 2 - Estruturação do método

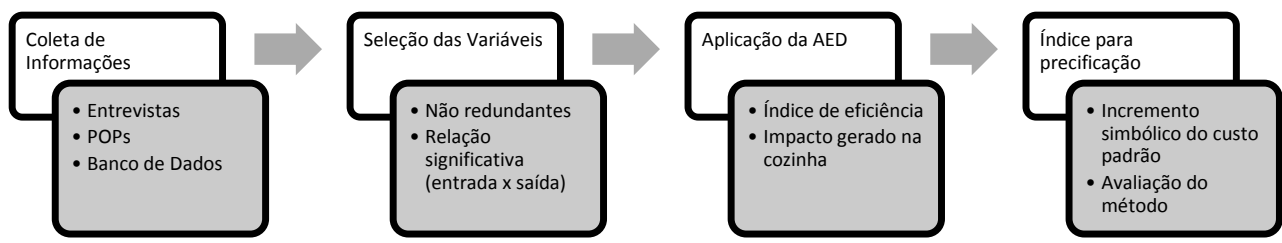

Fonte: Autoria própria (2016).

\section{COLETA DAS INFORMAÇÕES}

Os dados para a execução da análise originam-se de três fontes: entrevistas com a equipe gerencial, Procedimentos de Operação Padrão (POPs) e banco de dados do sistema. As entrevistas serviram para elencar os fatores que podem descrever a complexidade da preparação de cada prato. Optou-se por utilizar a equipe gerencial nesta etapa tendo em vista que seus integrantes possuem amplo conhecimento do processo, uma vez que foram operadores na cozinha antes de assumir os postos gerenciais.

A definição do impacto de cada fator levantado pela equipe gerencial no preparo de cada prato foi feita observando-se os POPs, que detalham o preparo de cada prato. Se o número de matérias primas necessárias para o preparo de um prato, por exemplo, for considerado um fator relevante pela equipe gerencial, a informação de quantas matérias primas são utilizadas em cada prato é levantada nos POPs. O banco de dados do sistema, por sua vez, foi utilizado para fornecer informações de vendas e tempo de preparo real dos pratos.

\section{SELEÇÃO DAS VARIÁVEIS}

A seleção das variáveis com potencial a serem inseridas na AED foi feita com o uso do software Microsoft Excel 2010. Optou-se pela utilização desse software pelo fato de o mesmo ser utilizado na empresa onde o estudo foi aplicado, possibilitando assim futuras replicações da análise e a efetiva utilização do método para precificação dos cardápios.

Verificou-se estatisticamente se as variáveis pré-selecionadas possuíam os requisitos necessários para serem utilizadas na Análise Envoltória de Dados. Inicialmente os dados foram normalizados para evitar a influência da magnitude das variáveis na seleção das mesmas. Em seguida, foi realizado um teste de correlação para a identificação das variáveis de entrada mais correlacionadas com as demais. Concomitantemente realizou-se um teste de regressão para verificar a relação entre as variáveis de entrada e saída e, na sequência, os resultados de ambos os testes foram relacionados para a seleção das variáveis.

O teste de correlação resultou no IACorr (índice acumulado de correlação), formado pelo somatório dos módulos das correlações das variáveis com as demais. O teste de regressão, por sua vez, gerou coeficientes de regressão (Coef) que representam a relação de cada variável com as de saída. Por fim, foi desenvolvido um índice composto pelo quociente Coef/IACorr. Variáveis com esse índice maior que a mediana foram consideradas adequadas, visto que, 
quanto maior o Coef e menor o IACorr, mais adequada é a variável para ser utilizada na AED. Assim, as variáveis de entrada que apresentaram maior relação com as variáveis de saída e menor correlação com as demais foram selecionadas. Esta etapa resultou, portanto, em uma seleção de variáveis não redundantes capazes de descrever a influência dos fatores críticos de produção de cada prato na geração das variáveis de saída.

\section{APLICAÇ̃̃O DA ANÁLISE ENVOLTÓRIA DE DADOS}

Após a seleção das variáveis, aplica-se a AED também com auxílio do software Microsoft Excel 2010. Nessa etapa, o Modelo CCR foi utilizado para a geração de índices que quantificam a eficiência de cada prato na transformação das variáveis de entrada em variáveis de saída. Essa relação pode ser vista também como o quanto cada prato onera a cozinha e estruturas de apoio ao ser preparado, uma vez que um prato mais eficiente utiliza menos recursos para ser produzido.

Para que os índices não refletissem elementos intrínsecos à preparação e à demanda natural de determinada categoria de pratos, a análise foi dividida em grupos de acordo com a classificação proposta pelo cardápio. Essa separação também é importante porque se supõe que o preço tem influência significativa na decisão do cliente após a escolha da categoria de prato que o mesmo irá consumir. Por exemplo, o cliente opta por um hambúrguer e, após tomar essa decisão, pode utilizar a informação do preço para decidir, dentre os hambúrgueres disponíveis, qual irá consumir.

\section{CRIACCÃO DE ÍNDICE PARA PRECIFICAÇÃO}

O objetivo desta etapa é transformar a informação de eficiência dos pratos, obtida com a aplicação da AED, em um índice que possa auxiliar na precificação. Uma vez que o método utilizado para precificar o cardápio consiste na observação do custo das matérias primas utilizadas em cada prato, esse índice foi criado em valor monetário e adicionado ao custo de alimento dos pratos. Os pratos menos eficientes são, portanto, penalizados com o acréscimo de um custo simbólico que representa o impacto negativo que geram na estrutura do restaurante ao serem solicitados pelo cliente. Dessa forma, esses pratos tendem a receber um preço maior do que os mais eficientes e, consequentemente, a demanda tende a se concentrar naqueles pratos que geram menor impacto para serem produzidos.

Em seguida, com o objetivo de avaliar a sistemática proposta, foram propostos cenários à equipe responsável pela precificação dos cardápios nos restaurantes. Os cenários simularam a precificação de pratos de uma determinada categoria com e sem o índice de precificação gerado.

\section{RESULTADOS}

Nesta seção são apresentados os resultados obtidos com a aplicação da metodologia proposta. Inicialmente apresentam-se as variáveis listadas como relevantes pela equipe gerencial. Após, são expostos os resultados da seleção 
dessas variáveis e, por sua vez, mostra a aplicação da AED. Na sequência, são apresentados os índices de precificação criados e o impacto dos mesmos na precificação. Por fim, é realizada uma discussão acerca do método de precificação proposto.

\section{COLETA DAS INFORMAÇÕES}

A realização de entrevistas com a equipe gerencial dos restaurantes resultou em sete variáveis com potencial para refletir a demanda por recursos do restaurante gerada por cada prato:

a) recursos utilizados (I): número total de recursos da cozinha utilizados para o preparo do prato (ex. chapa, grelha, micro-ondas, gratinador, etc.);

b) tempo de uso dos recursos (II): somatório do tempo de utilização dos recursos envolvidos na montagem do prato;

c) componentes (III): quantidade de componentes usados na montagem do prato;

d) áreas ativadas (IV): número de áreas da cozinha (chapa, grelha, fritadeira e montagem final) ativadas para o preparo de um prato;

e) MPs total (V): somatório de todas as matérias primas utilizadas no preparo do prato;

f) MPs exclusivas (VI): número de matérias primas utilizadas apenas no prato em questão;

g) preps exclusivas (VII): quantidade de preps (alimentos pré-prontos para a montagem final) que são exclusivas do prato em questão.

Percebe-se que as variáveis (I), (II), (III) e (IV) descrevem o impacto que o prato causa na cozinha ao ser solicitado pelo cliente, pois refletem a demanda por estrutura física e mão de obra gerada pelo mesmo. As variáveis (V), (VI) e (VII), por sua vez, estão relacionadas com as atividades de apoio necessárias para que o prato seja produzido (compras, controle de estoques, produção de preps, etc.).

Nesta etapa concluiu-se também que o número de unidades vendidas é o parâmetro mais adequado para descrever o sucesso de determinado prato. Assim, foram levantadas as vendas de cada prato no período de um ano para a geração das variáveis de saída.

\section{SELEÇÃO DAS VARIÁVEIS}

Os resultados da análise da correlação existente entre as variáveis de entrada podem ser visualizados na Tabela 1 . Com base nos resultados dessa análise obteve-se ao IACorr (índice acumulado de correlação), que é o somatório dos módulos das correlações de cada variável de entrada com as demais. Uma vez que não se deseja a existência de correlação entre as variáveis de entrada (requisito do $\mathrm{AED}$ ), quando menor for o IACorr da variável, mais adequada será a mesma para a utilização na AED. 
Tabela 1 - Análise de correlação entre as variáveis de entrada

\begin{tabular}{|c|c|c|c|c|c|c|c|}
\hline$\frac{\sqrt{10}}{\frac{\sqrt{10}}{10}}$ & 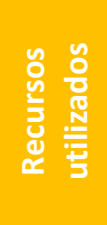 & 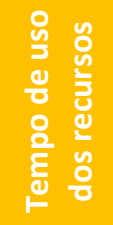 & 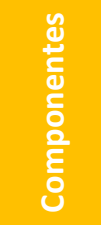 & 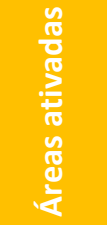 & 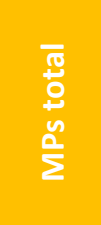 & 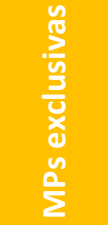 & 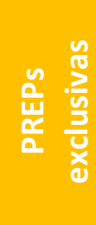 \\
\hline $\begin{array}{l}\text { Recursos } \\
\text { utilizados }\end{array}$ & 1,000 & 0,598 & 0,269 & 0,930 & 0,195 & 0,176 & 0,027 \\
\hline $\begin{array}{l}\text { Tempo de uso dos } \\
\text { recursos }\end{array}$ & 0,598 & 1,000 & 0,350 & 0,648 & 0,192 & 0,161 & $-0,031$ \\
\hline Componentes & 0,269 & 0,350 & 1,000 & 0,376 & 0,720 & 0,126 & 0,139 \\
\hline Áreas ativadas & 0,930 & 0,648 & 0,376 & 1,000 & 0,272 & 0,136 & 0,000 \\
\hline MPs total & 0,195 & 0,192 & 0,720 & 0,272 & 1,000 & 0,037 & 0,071 \\
\hline MPs exclusivas & 0,176 & 0,161 & 0,126 & 0,136 & 0,037 & 1,000 & 0,384 \\
\hline PREPs exclusivas & 0,027 & $-0,031$ & 0,139 & 0,000 & 0,071 & 0,384 & 1,000 \\
\hline IACorr & 3,195 & 2,981 & 2,980 & 3,362 & 2,488 & 2,020 & 1,653 \\
\hline
\end{tabular}

Fonte: Autoria própria (2016).

A aplicação do modelo de regressão resultou nos coeficientes de regressão (Coef). Esses coeficientes quantificam a relação entre as variáveis de entrada e a variável de saída. Quanto maior o módulo de Coef, maior é a influência da variável de entrada na determinação da variável de saída. Para evitar a influência da magnitude dos valores na seleção das variáveis, tanto os valores de Coef como os do IACorr foram escalonados em uma escala de 0 a 1, gerando os coeficientes Coef $_{\text {esc }}$ e IACorr esc

A definição das variáveis que permaneceram na análise foi feita com a criação de um índice composto pelo quociente entre o módulo do Coef esc e o IACorr $_{\text {esc }}$. Esse índice relaciona a correlação existente entre determinada variável de entrada e as demais com a influência da mesma na variável de saída. Quanto maior for o índice, menos correlacionada com as demais variáveis de entrada e/ou mais relacionada com a variável de saída estará a variável em análise. Portanto, consideraram-se as variáveis adequadas para o uso na AED aquelas com o índice Coef esc $_{\text {/ IACorr }}$ esc superior à mediana dos quocientes. A Tabela 2 apresenta os resultados dessa análise, que resultou nas variáveis selecionadas para a execução da AED: recursos utilizados, áreas ativadas e MPs total. 
Tabela 2 - Avaliação das variáveis

\begin{tabular}{|c|c|c|c|c|}
\hline Variável & Coef $_{\text {esc }}$ & IACorr & Coef $_{\text {esd }} /$ IACorr $_{\text {esc }}$ & Classificação \\
\hline Recursos utilizados & 0,556 & 0,950 & 0,585 & Dentro \\
\hline Tempo de uso dos recursos & 0,038 & 0,868 & 0,044 & Fora \\
\hline Componentes & 0,109 & 0,887 & 0,123 & Fora \\
\hline Áreas ativadas & 1,000 & 1,000 & 1,000 & Dentro \\
\hline MPs total & 0,140 & 0,740 & 0,190 & Dentro \\
\hline MPs exclusivas & 0,093 & 0,601 & 0,156 & Dentro \\
\hline \multirow[t]{2}{*}{ PREPs exclusivas } & 0,019 & 0,473 & 0,040 & Fora \\
\hline & & Mediana & 0,156 & \\
\hline
\end{tabular}

Fonte: Autoria própria (2016).

\section{APLICAÇÃO DA ANÁLISE ENVOLTÓRIA DE DADOS}

A aplicação da AED foi feita com a utilização das variáveis de entrada selecionadas na etapa anterior e com a variável de saída (vendas), definida na etapa 4.1. A AED foi aplicada para todos os pratos do cardápio. Este estudo, porém, se limitará a analisar os resultados de uma categoria de produtos, visto que a sistemática adotada para as demais é idêntica.

Os resultados da AED na Categoria A são apresentados na Tabela 3. Apenas o Prato 7 atingiu a relação ótima de transformação dos inputs em outputs, classificando-se como uma DMU $100 \%$ eficiente. Os demais pratos tiveram suas eficiências determinadas com base nessa DMU, sendo o Prato 10 mais ineficiente, apesar de não ser aquele com menor venda.

Tabela 3 - Resultados da aplicação da AED na categoria Carnes

\begin{tabular}{ccccccc} 
Categoria A & $\begin{array}{c}\text { MPs } \\
\text { total }\end{array}$ & $\begin{array}{c}\text { Recursos } \\
\text { utilizados }\end{array}$ & $\begin{array}{c}\text { MPs } \\
\text { exclusivas }\end{array}$ & $\begin{array}{c}\text { Áreas } \\
\text { ativadas }\end{array}$ & $\begin{array}{c}\text { Vendas } \\
(\times 1000)\end{array}$ & $\begin{array}{c}\text { Eficiência } \\
\%\end{array}$ \\
Prato 1 & 12 & 3 & 0 & 5 & 4,282 & 25,02 \\
Prato 2 & 12 & 2 & 0 & 4 & 4,395 & 38,53 \\
Prato 3 & 7 & 2 & 0 & 3 & 3,075 & 29,95 \\
Prato 4 & 27 & 3 & 0 & 5 & 9,756 & 57,02 \\
Prato 5 & 30 & 2 & 0 & 4 & 5,018 & 43,99 \\
Prato 6 & 10 & 2 & 1 & 4 & 5,230 & 45,85 \\
Prato 7 & 9 & 3 & 0 & 5 & 17,111 & 100,00 \\
Prato 8 & 9 & 3 & 0 & 5 & 8,485 & 49,59 \\
\hline
\end{tabular}

Fonte: Autoria própria (2016).

A AED foi aplicada com a resolução de oito Problemas de Programação Linear (PPLs), que geraram pesos v1, v2, v3, v4 e u1 capazes de maximizar as eficiências de cada variável sem que as demais ultrapassem $100 \%$. Visto que foram encontradas diversas soluções ótimas para cada PPL, pode-se afirmar que existem infinitas soluções para os mesmos. Uma dessas soluções, que atende às restrições impostas pela formulação da AED e maximiza as eficiências das DMUs, é apresentada na Tabela 4. Cada prato possui um peso u1 que, multiplicado pela 
variável de saída, gera o índice de eficiência do prato. Os pesos v1, v2, v3 e v4, por sua vez, são multiplicadores das variáveis de entrada.

Tabela 4 - Possíveis soluções dos PPLs que compõem a AED

\begin{tabular}{ccccccc} 
Categoria A & $v 1$ & v2 & $v 3$ & $v 4$ & u1 \\
Prato 1 & 0,000 & 0,333 & 0,000 & 0,000 & 0,058 \\
Prato 2 & 0,000 & 0,500 & 0,000 & 0,000 & 0,088 \\
Prato 3 & 0,000 & 0,000 & 0,000 & 0,333 & 0,097 \\
Prato 4 & 0,000 & 0,333 & 0,000 & 0,000 & 0,058 \\
Prato 5 & 0,000 & 0,500 & 0,000 & 0,000 & 0,088 \\
Prato 6 & 0,000 & 0,500 & 0,000 & 0,000 & 0,088 \\
Prato 7 & 0,000 & 0,000 & 1,000 & 0,200 & 0,058 \\
Prato 8 & 0,000 & 0,000 & 25,000 & 0,200 & 0,058 \\
\hline
\end{tabular}

Fonte: Autoria própria (2016).

\section{CRIAÇÃO DE ÍNDICE PARA PRECIFICAÇÃO}

O índice de precificação foi criado para penalizar os pratos que apresentaram eficiência menor que a média, aumentando simbolicamente os custos reais de matéria prima desses pratos. A Tabela 5 apresenta a escala utilizada para esses aumentos, que varia de $0 \%$ a $50 \%$, visto que a média das eficiências é igual a $48,75 \%$. Já o valor de $20 \%$ para o aumento máximo dos custos foi definido com base na variação de preços que a empresa considera aceitável para não prejudicar a imagem do estabelecimento perante os clientes.

Tabela 5 - Escala de aumento dos custos com base na eficiência

\begin{tabular}{cccc} 
& Eficiência & & Aumento \\
$0 \%$ & - & $10 \%$ & $20 \%$ \\
$10 \%$ & - & $20 \%$ & $16 \%$ \\
$20 \%$ & - & $30 \%$ & $12 \%$ \\
$30 \%$ & - & $40 \%$ & $8 \%$ \\
$40 \%$ & - & $50 \%$ & $4 \%$ \\
& $>50 \%$ & & $0 \%$ \\
\hline
\end{tabular}

Fonte: Autoria própria (2016).

A avaliação do impacto que o aumento simbólico dos custos pode ter na precificação foi avaliada através da simulação de cenários, propostos pelos responsáveis pela determinação dos preços nos restaurantes. O Cenário 1, cujos resultados estão dispostos na Tabela 6, propôs a determinação dos preços com base nos custos reais de cada prato. Para evitar a influência de fatores subjetivos, foi informado apenas de qual categoria se tratavam os pratos, não especificando o nome dos mesmos. A equipe determinou então o preço dos pratos com base na informação do custo e das margens brutas individuais, simuladas instantaneamente com base nos preços líquidos (sem impostos). 
Tabela 6 - Cenário 1: custos reais

\begin{tabular}{ccccc} 
Categoria A & Custo $\$$ & $\begin{array}{c}\text { Margem bruta } \\
\text { Margem }\end{array}$ & $\begin{array}{c}\text { Mareço } \\
\text { bruta } \%\end{array}$ \\
Prato 1 & 13,94 & 29,77 & 68,1 & 49,90 \\
Prato 2 & 8,21 & 22,01 & 72,8 & 34,50 \\
Prato 3 & 9,33 & 24,40 & 72,3 & 38,50 \\
Prato 4 & 12,02 & 31,69 & 72,5 & 49,90 \\
Prato 5 & 10,54 & 27,92 & 72,6 & 43,90 \\
Prato 6 & 11,02 & 29,19 & 72,6 & 45,90 \\
Prato 7 & 14,03 & 29,68 & 67,9 & 49,90 \\
Prato 8 & 9,98 & 25,85 & 72,1 & 40,90 \\
\hline
\end{tabular}

Fonte: Autoria própria (2016).

$\mathrm{Na}$ sequência, foram aplicados os aumentos sugeridos pela Tabela 5 para o custo de cada prato de acordo com as suas eficiências. Gerou-se então o Cenário 2 , com os novos custos, que também foi proposto à equipe do restaurante para a precificação. A Tabela 7 mostra os preços resultantes desse cenário, assim como o aumento que foi aplicado aos custos e a diferença dos preços em relação ao Cenário 1.

Tabela 7 - Cenário 2: custos acrescidos de índice com base na eficiência

\begin{tabular}{ccccccc} 
Categoria A & Custo $\$$ & $\begin{array}{c}\text { Margem } \\
\text { bruta } \$\end{array}$ & $\begin{array}{c}\text { Margem } \\
\text { bruta \% }\end{array}$ & Preço \$ & $\begin{array}{c}\text { Aumento } \\
\text { custo \% }\end{array}$ & $\begin{array}{c}\text { Aumento } \\
\text { preço \% }\end{array}$ \\
\hline Prato 1 & 15,61 & 30,73 & 66,3 & 52,90 & 12 & 6 \\
Prato 2 & 8,87 & 22,58 & 71,8 & 35,90 & 8 & 4 \\
Prato 3 & 10,45 & 27,13 & 72,2 & 42,90 & 12 & 11 \\
Prato 4 & 12,02 & 31,69 & 72,5 & 49,90 & 0 & 0 \\
Prato 5 & 10,96 & 28,37 & 72,1 & 44,90 & 4 & 2 \\
Prato 6 & 11,46 & 29,62 & 72,1 & 46,90 & 4 & 2 \\
Prato 7 & 14,03 & 29,68 & 67,9 & 49,90 & 0 & 0 \\
Prato 8 & 10,38 & 26,85 & 72,1 & 42,50 & 4 & 4 \\
\hline
\end{tabular}

Fonte: Autoria própria (2016).

\section{IMPLICAÇÕES GERENCIAIS DOS RESULTADOS}

Em ambos os cenários, a equipe de precificação buscou manter a margem bruta percentual próxima dos $72 \%$. Isso ocorre porque se considera $70 \%$ de margem o número adequado para o setor em que o restaurante trabalha, sendo os $2 \%$ excedentes adicionados como margem de segurança para as perdas normais do processo. Consequentemente, todos os pratos que tiveram um aumento simbólico gerado pelo índice de precificação também tiveram os seus preços de venda propostos aumentados. O índice de precificação teve, portanto, êxito ao induzir aumentos nos valores dos pratos menos eficientes. A demanda dos pratos não acrescidos do índice de precificação tende a ser maior, onerando assim uma menor estrutura do restaurante para a venda do mix de produtos proposto. 
No entanto, o efeito da replicação do método nas precificações seguintes pode torná-lo limitado. Visto que a eficiência dos pratos depende da quantidade vendida dos mesmos, quanto maior a venda do prato, mais eficiente ele será e vice-versa. Assim, os pratos com menos venda - e possivelmente menos eficientes - terão as vendas diminuídas ainda mais devido ao aumento de preço proposto pela metodologia. Essa diminuição nas vendas tornará o prato ainda mais ineficiente, resultando em um preço ainda maior. Cria-se então um "espiral da morte", no qual os pratos ineficientes tendem a ser eliminados do cardápio.

Observou-se ainda que alguns fatores subjetivos influenciaram significativamente na precificação, mesmo sem que a equipe tivesse conhecimento dos pratos que estavam sendo precificados. Tomou-se o cuidado, por exemplo, de evitar que os pratos ultrapassassem o valor de $\mathrm{R} \$ 50,00$, considerado o teto para a categoria em análise. A determinação dos "valores quebrados" também permaneceu sem uma lógica clara, sendo os centavos determinados de acordo com o padrão que habitualmente é utilizado.

\section{CONCLUSÕES}

O estudo propôs a utilização da AED para aprimorar a sistemática de precificação de cardápios em um restaurante casual dining. Visto que os modelos tradicionais consideram apenas o custo dos ingredientes na determinação dos preços, criaram-se índices de eficiência capazes de ponderar na precificação o impacto da produção dos pratos na cozinha. Os índices foram criados com base nos resultados da AED e penalizam com um valor monetário simbólico os pratos que oneram mais recursos do restaurante ao serem produzidos.

Cenários com os custos de ingredientes foram apresentados à equipe de precificação para simular a aplicação da metodologia proposta. Os preços de $75 \%$ dos pratos foram maiores com os custos ajustados, mostrando que a sistemática é eficiente em considerar na precificação a utilização dos recursos do restaurante necessária para a produção de cada prato. Visto que a demanda pelos pratos penalizados tende a diminuir, o mix de vendas será concentrado nos produtos mais eficientes, que utilizam menos recursos do restaurante. Assim, espera-se que ocorram ganhos operacionais com o uso da sistemática.

Ressalta-se, porém, que a utilização da AED para a precificação de cardápios deve ser feita com cautela. Uma vez que a variável do número de vendas de cada produto possui forte impacto na determinação da eficiência do prato, os pratos com menores vendas tendem a ser menos eficientes e, consequentemente, terem os preços aumentados após a precificação. $O$ incremento nos preços irá reduzir a demanda desses pratos, tornando-os ainda menos eficientes. Cria-se então uma espiral da morte em que os pratos não eficientes tendem a ser eliminados do cardápio. 


\title{
Data envelopment analysis for princing improvement in a casual dining restaurant
}

\begin{abstract}
The high competitiveness in different area incites companies to find solutions to differentiate themselves from the competition. In the food area the profitability is related to product prices, making pricing process important in the strategic planning of companies. This paper proposes a Data Envelopment Analysis (EDA) based method for improving pricing process in a casual dining restaurant. For that matter, indices measuring the resources used in each item on the menu were generated. Such indices enabled assessing the efficiency of each menu item in terms of resources and price, providing means to further decision regarding pricing. Different cost scenarios were simulated to assess the influence of such index on the pricing process. When applied to a restaurant menu, the method provided managers with a reliable framework to price menu items other than costs of ingredients.
\end{abstract}

KEYWORDS: Menu engineering. Pricing. Data envelopment analysis. Casual dining restaurant. Items efficiency. 


\section{REFERÊNCIAS}

ALVAREZ F. E.; LIPPI, F.; PACIELLO, L. Optimal price setting with observation and menu costs. Quarterly Journal of Economics, p. 1909-1960, 2011. crossref

AIGNER, D. J.; LOVELL, C. A. K.; SCHMIDT, P. Formulation and estimation of stochastic frontier production function models. Journal of Econometrics, v. 6, p. 21-77, 1977. crossref

BELLONI, I. Uma metodologia de avaliação da eficiência produtiva de universidades federais brasileiras. 2000. Tese (Doutorado em Engenharia de Produção) - Universidade Federal de Santa Catarina. Florianópolis, 2000.

CARMIN, J.; NORKUS, G. X. Pricing strategies for menus: magic or myth? The Cornell Hotel and Restaurant Administration Quarterly, v. 31, p. 44-50, 1990. crossref

CHARNES, A.; COOPER, W. W.; RHODES E. Measuring the efficiency of decisionmaking units. European Journal of Operational Research, v. 2, p. 429-444, 1978. crossref

CHARNES, A.; COOPER, W. W. Programming with linear fractional funcionals. Naval Research Logistics, p. 181-186, 1962. crossref

CHOU, S. F.; FANG, C. Y. Exploring surplus-based menu analysis in Chinese-style fast food restaurants. International Journal of Hospitality Management, v.33, p. 263-272, 2013.

COOPER, W. W.; SEIFORD, L. M.; ZHU, J. Handbook on data envelopment analysis. Springer Science \& Business Media, 2011.

COELLI, T. J. Recent developments in frontier modeling and efficiency measurement. Australian Journal of Agricultural Economics, v. 39, p. 219-245, 1995. crossref

GIL, A. Como elaborar projetos de pesquisa. 4. ed. São Paulo: Atlas, 2008.

HAYES, D. K.; HUFFMAN, L. M. Value pricing: how long can you gou? The Cornel Hotel and Restaurant Administration Quarterly, v. 36, 1995. 
HSIEH, L. F.; LIN L. H. A performance evaluation for international tourist hotels in Taiwan: an application of the relational network DEA. International Journal of Hospitality Management, v. 29, p. 14-24, 2010. crossref

KASAVANA, M. L.; SMITH, D. I. Menu engineering: a practical guide to menu analysis. Hospitality Publications, 1982

KIEFER, N. M.; KELLY, T. J.; BURDETT, K. Menu pricing: an experimental approach. Journal of Business \& Economical Statistics, v. 12, p. 329-337, 1994. crossref

KELLY, T. J.; KIEFER, N. M.; BURDETT, K. A demand-based approach to menu pricing. The Cornell H.R.A. Quarterly, v. 50, p. 383-387, 2009.

KWONG, L. Y. L. The application of menu engineering and design in Asian restaurants. International Journal of Hospitality Management, v. 24, p. 91-106, 2005. crossref

LINASSI, R. Engenharia de cardápio e custeio baseado em atividades. 2009. Dissertação (Mestrado em Turismo e Hotelaria) - Universidade do Vale do Itajaí. Balneário Camboriú, 2009.

MELLO, J. C. C. B. S.; et al. Curso de Análise Envoltória de Dados. In: SIMPÓSIO BRASILEIRO DE PESQUISA OPERACIONAL, v. 37, 2005, Gramado. Anais... Gramado, 2005.

MELLO, J. C. B. S.; et al. Análise envoltória de dados no estudo da eficiência e dos benchmarks para companhias aéreas brasileiras. Pesquisa Operacional, v. 23, p. 325-343, 2003. crossref

MEEU SEN, W.; VAN DEN BROECK, J. Efficiency estimation from Cobb-Douglas production functions with composed error. International Economic Review, v. 18 , p. 435-444, 1977. crossref

MILLER, J. E. Menu pricing and strategy. CBI Publishing Company. 1980.

PAVESIC, D. V. Cost-margin analysis: a third approach to menu pricing and design. International Journal of Hospitality Management, v. 2, n. 3, p. 127-134, 1983. crossref

PAVESIC, D. V. Psychological aspects of menu pricing. International Journal of Hospitality Management, v. 8, p. 43-49, 1989. cross ref 
RAAB, C.; MAYER, K. Menu engineering and activity-based costing - can they work together in a restaurant? International Journal of Contemporary Hospitality Management, v. 19, n. 1, p. 43-52, 2007. crossref

REYNOLDS, D.; TAYLOR, J. Validating a DEA-based menu analysis model using structural equation modeling. International Journal of Hospitality Management, v. 30 , p. 584-587, 2011. crossref

REYNOLDS, D.; THOMPSON, G. M. Multiunit restaurant productivity assessment using three-phase data envelopment analysis. International Journal of Hospitality Management, v. 26, p. 20-32, 2007. crossref

SOON, W. A review of multi-product pricing models. Applied Mathematics and Computation, v. 217, p. 8149-8165, 2011. crossref

TAYLOR, J.; REYNOLDS, D.; BROWN D. Multi-factor menu analysis using data envelopment analysis. International Journal of Contemporary Hospitality Management, v. 21, n. 2, p. 213-225, 2009. crossref

YANG, S. S.; KIMES, S. E.; SESSAREGO, M. M. Menu price presentation influences on customer purchase behavior in restaurants. International Journal of Hospitality Management, v. 28, p. 157-160, 2009. crossref 
Recebido: 06 jul. 2016

Aprovado: 01 fev. 2017

DOI: 10.3895/gi.v13n1.4428

Como citar:

SUSIN, G. et al. Análise envoltória de dados para aprimorar a sistemática de precificação em um restaurante casual dining. R. Gest. Industr., Ponta Grossa, v. 13, n. 1, p. 1-21, jan./mar. 2017. Disponível em: <https://periodicos.utfpr.edu.br/rgi >. Acesso em: XXX.

Correspondência:

Giovanni Susin

Av. Osvaldo Aranha, 99, $5^{\circ}$ andar, Porto Alegre, Rio Grande do Sul, Brasil.

Direito autoral: Este artigo está licenciado sob os termos da Licença Creative Commons-Atribuição 4.0 Internacional.

(c) (1) 